\title{
Psychodemographic Factors Predicting Internet Fraud Tendency among Youths in Southwestern, Nigeria
}

\author{
Elgbadon E. Gregory PhD \\ Department of Psychology, University of Ibadan, Nigeria \\ Adejuwon A. Grace PhD \\ Department of Psychology, University of Ibadan, Nigeria
}

\section{Doi:10.5901/jesr.2015.v5n2p159}

\section{Abstract}

Advancement in telecommunications globally within the last two decades has consistently been accompanied with an upsurge in incidences of e-fraud. The objective of this study was to investigate the psychological and demographic factors such as peer influence, materialistic value, impulsivity, self-control, age, sex and highest educational factors as they predict internet fraud tendency among youths that patronise cybercafés in south-western Nigeria. The study utilized a cross-sectional survey with an ex-post facto design, 986 youth internet users from Lagos and Ibadan were purposively sampled to participate in the study. The factors jointly predicted internet fraud tendency among internet users $(R 2=.23, F(7,965)=40.684 .76, P<.001)$. Independently, peer influence and materialistic value $(\beta=-.096, \quad t=-2.97, \quad p<.01 ; \beta=-.396 t=-12.24, p<.001)$ were significant. In the $23 \%$ variation observed in the prediction, peer influence contributed $19.5 \%$ while materialistic values, age and sex contributed $3.5 \%$. Therefore, the development of intervention programmes for mitigating factors that predispose youths to internet fraud practices should include counselling on keeping the right company, resisting peer pressure and placing less emphasis on material acquisition

Keywords: Peer-influence, internet-use, internet fraud tendency.

\section{Introduction}

Internet fraudulent activities have been on a consistent increase globally (Osimiri, 1997; Buchanam, 2001;Tanfa, 2006). The preference for the use of internet medium to defraud unsuspecting victim among other reasons is that, there is an opportunity to make vast number of calls or e-mails at ridiculously low cost with low barriers to entry and extended geographical reach. It is also related to the fact that the proceeds from the crime are secured anonymously and in jurisdiction where the pursuit of the offender is difficult. The prospective victims are also conditioned to believe what appears on a computer screen. In comparison to other forms of crime, these characteristics make Internet fraud interesting and useful for academic study (Chua \& Wareham, 2002, 2004; Grazioli \& Javenpaa, 2003). Given these aforementioned reasons for using the Internet to defraud, it should be noted however, that it is not every youth with a good knowledge of computer that is using it to defraud. In other words what are the salient factors that differentiate youths perpetuating internet fraud from those who are not? It is important to contribute to existing literature by exploring factors promoting these tendencies among youths in Nigeria.

Adeniran (2006) observed that the Internet has brought an astounding alteration in the behavioural patterns of Nigerian youths. The 'modernization of criminality' through the electronic superhighway has been serving as the platform for the emergence and sustenance of fraudulent sub-culture in Nigeria. This view is corroborated by Rogers, Siegfried and Tidke (2006) who noted that individuals (17-30 years) anecdotally make up the majority of those individuals engaged in deviant or criminal behaviour.

Gottfredson and Hirschi (1990) in their "General Theory of Crime" posit that when unrestrained individual (Persons with low self-control) encounters opportunities for gratifying but socially undesirable behaviour, they are most likely to act on them. Foster (2004) also support this view that low self-control underlies most of human behaviour or pathological tendencies. Tangney, Baumeister and Boone (2004) noted that central to the concept of self control is the ability to override or change one's inner responses as well as to interrupt undesired behavioural tendencies and refrain from acting on them. The assumptions of Gottfredson and Hirschi (1990) on criminal behaviour which Tangney, Baumeister and 
Boone (2004) also corroborated can also be said to reflect in other people who may not have been apprehended for fraudulent behaviour.

The adoption of materialistic values by young people also affects the balance between the private (internal) and public (external) choices that youth make throughout life (Golberg, Gorn, Peracchis \& Bomossy, 2003). These choices which are often influenced by a high tendency for material acquisition may also reflect the possibility of perpetuating fraudulent behaviour. Materialism is an orientation that ascribes much importance to the acquisition of material possessions; but also implies a belief that possessions are a means to greater happiness and life satisfaction, as well as a measure of success and a tool for social comparison. Materialism has been treated as a negative value, connected to possessiveness, envy, lack of generosity, greed and jealousy (Belk, 1983). When individual's psychological needs are not met, they may tend to move toward materialism as a type of compensatory strategy to lessen the distressing effects of feelings of insecurity. Individuals also learn to adopt materialistic values through social learning from family members, peers, and the materialistic messages frequently found in television programs and commercials Kasser, Ryan, Couchman and Sheldon (2004).In a longitudinal study by Easterlin and Crimmins (1991) on high school students from early 1970's to 1980's there were indications of sharp increase in private materialism as a life goal and a sharp decline in emphasis on personal self-fulfilment. Burroughs and Rindfleisch (2002) reported high level of material value has been found to create tension between the individual orientation towards material values and a collective orientation towards family and religious values.

Another psychological explanation for internet fraud tendency is peer pressure.Youths often prefer to identify with their peer groups. This relate to the extent to which a youth is susceptible to influence from friends or significant others. Chan and Zhang (2007) noted that peer group with shared experience are an inevitable source of personal relationships. The frequent interactions with peers, particularly with deviant peers sometimes lead to the adoption of antisocial behaviour for group conformity. Young people need to formulate a new identity and to establish autonomy from their parents. The frequent interaction with peers, even more frequent than with parents, can lead to peers becoming the primary basis for social comparison. A generalized social comparison theory states that individuals compare their own material possessions with those owned by significant others to ascertain their social status (Saunders, 2001). However, many research indicated that deviant peers had less influence on adolescents who had close relationships with their parents (Coombs, Paulson, and Richardson, 1991; Rankin \& Kern, 1994).

Atwai (2011) observed that Peer influence and low self-control appear to be the major factors fuelling juvenile cybercrime such as computer hacking and online bullying. He noted that having friends who engage in cybercrime is one of the greatest determinants in whether juveniles will commit cybercrimes. In another related study, Holt (2011) administered a scientific survey to 435 students in a Kentucky school district. Study results showed that the biggest predictor that juveniles might engage in cybercrime is peer influence. He also observed that low self-control have both a direct and indirect effect, through other peers offending, on youth cybercrime. The study notes that both low self-control and deviant peer associations have been linked, not only to cybercrime violations, but also to committing other crimes. They also found that peer offending had a stronger effect on others offending than low self-control and also consistently predicted each type of cyber deviance. In the study, it was noted that cybercrimes may be more attractive to youths than real world offenses because of the relative anonymity that the Internet and computers provide their users.

Burton, Evans, Cullen, Olivares, and Dunaway (1999) observed that the younger a youth's age, the more imprudent behaviour they perpetrate among youths (18-30 years). This observation has implication for internet fraud practices. Age positively correlated with cybercrime, Females were very unlikely to commit cybercrimes. Levels of Education have also been determined to be significant in the manifestation of criminal behaviour. School achievements are predictive of pro-social behaviour or behaivour's designated as upholding the moral values of a society. This is because academic achievement is interrelated in our society with several other variables such as financial success, high self-esteem and an internal locus of control Jackson et al (1985). This particular model may account for reasons behind the general idea that individuals with high Intelligent Quotient (IQ) generally have fewer tendencies for criminal behaviour than with a low IQ Blackburn (1995).

The growing concern on internet fraudulent activities need to be addressed at the policy making level in Nigeria. Conclusively, data based information on factors associated with cybercrimes among youths need to be provided to inform policy and implementation in Nigeria. To address these issues in Nigeria and specifically within the south western region, the current study examined whether psychological factors such as self-control, peer influence, materialistic values and impulsivity will significantly independently and jointly predict internet fraudulent tendencies among youths in south western Nigeria. It will also find out whether psychological factors such as self-control, peer influence, materialistic values impulsivity and demographic factors such as age, sex and education will significantly independently and jointly 
predict internet fraudulent tendencies among youths in South-western Nigeria.

\section{Hypothesis}

Psychological factors such as self-control, peer influence, materialistic values impulsivity and demographic factors such as age, sex and education will significantly independently and jointly predict internet fraudulent tendencies among youths in South-western Nigeria

\section{Methods}

A cross-sectional research approach with an ex-post facto design (i.e. after-the-effect) was utilized among purposively selected youths that visit cyber cafes in south-western Nigeria. The study was limited to Lagos and Ibadan as both cities have heterogeneous population and is reflective of most commercial cities in the entire nation. Both cities are university towns where internet fraud activities are most likely to take place (Zero Tolerance, 2010).

\subsection{Procedure}

A pilot study with 120 participants was conducted in another location in Ibadan. The essence is to revalidate the existing instruments used in the study to prevent the introduction of error into the measurement since the settings in which they were previously used was different. At the completion of the pilot study, the data was analyzed and the coefficient alpha of all the measures was obtained before proceeding to the main study with other sets of participants who were not part of the initial revalidation process. 1200 questionnaires was purposively administered in the course of the study, only 986 $(82.16 \%)$ were retrieved.Among the respondents 411 (41.7\%) were female while 575 (58.3\%) were male. Age range is 15-29, $\bar{X}=23.58, \mathrm{SD}=4.04$.

\subsection{Instrument}

Demographic factors in the study includes, age, sex, and educational level of the respondents.

\subsection{Internet Fraud Tendency Scale}

This is an instrument developed by the researcher for this study. It is a 23-item scale generated from the outcome of indepth interview conducted for some youth samples $\mathrm{N}=7$ at various Cyber-Cafes in Ibadan, 2 Officials from the Economic and Financial Crime and Control (EFCC) were also interviewed. Focus Group Discussions (FGD) was conducted at 3 Cyber Cafes within the city. 9 participants were involved in each of the FGD's which held at 3 different locations in Ibadan. Content (face) validity of the scale was sort among 5 lecturers from the Department of Psychology, University of Ibadan. Examples of items in the scale includes, "If you are unemployed, the internet could be a means of assisting yourself", "It is not bad if you use your knowledge of internet to collect items from unsuspecting people", "Waiting for what the future holds is a waste of time when you can easily use the internet to become rich", Items in which the respondents were unanimous were retained as part of the items for the internet fraud tendency scale. The scale is uni-dimensional. Item loading ranges from .45 to .80 . The items loading of .45 was included because it satisfies the criterion for accepting structure coefficient (Pedhazur, 1982). Response ranges from Strongly Agree [5] Agree [4] Undecided [3] Disagree [2] Strongly Disagree [I].Guttman split-half reliability coefficient of .83 and Spearman Brown coefficient of .86 was obtained. High score indicates that the youth has the tendency to be involved in internet fraud if the opportunity presents itself. While, low score is indicative of either low or none internet fraud tendency. The scale had a $\bar{X}=19.12, \mathrm{SD}=4.15$. As part of the process for establishing the reliability of this scale, youths $\mathrm{N}=120$ ages between $15-29$ years participated.

\subsection{Self-Control Inventory}

This is a 36-item scale developed by Tangney et al [2004]. Items are rated on a 5-point likert scale anchored from 1 (not at all like me) to 5 (very much like me). The original scale had a reliability coefficient alpha of 0.93 . Revalidating the scale for this study, a reliability coefficient alpha $0.90, \mathrm{~N}=120$. A split-half reliability of 0.87 was obtained. 


\subsection{Materialistic value scale}

This is a shortened 6-item version suggested by Rinchins (2004). The inter-item reliability (Cronbach's Alpha) was 0.76 . Revalidation of the scale yielded a reliability of 0.69 and a Guttman Split-half coefficient of $0.58, N=120$. High score on this scale is indicative of a higher tendency towards materialistic values.

\subsection{Susceptibility to Peer Influence Scale}

This scale was originally developed by Mangleburg and Bristol (1998). It is a 6 item scale that considers the extent to which an individual's decision can be influenced by his or her peers. The inter-item reliability of the scale is 0.71 . adapting the scale and revalidation yielded a Cronbach Alpha of 0.78 and a Guttman Split-half coefficient of $0.83, \mathrm{~N}=120$.

\subsection{Impulsivity Scale}

This is an adapted version of Barratt Impulsivity scale (BIS-II). It is the most recent version of the scale. Developed in 1995, it is a widely used measure of self-administered impulsivity test. The scale has 30 items that are scored in a likert format ranging from always to rarely. Stanford and Barratt (1995) reported internal consistency coefficients for the scale that ranged from 0.79 to 0.83 for separate populations of undergraduate, substance abuse patients and prison inmates. Revalidation of the scale yielded a Chronbach alpha of 0.84 and a Guttman coefficient of $0.85, \mathrm{~N}=120$.

\section{Results}

The result section shows the description of all the variables and their prediction of internet fraud tendency among youths in lagos and Ibadan, Southwest Nigeria.

Table 1. Showing the descriptive statistics for variables in the study.

\begin{tabular}{|l|c|c|c|}
\hline Variables & N & Mean & SD \\
\hline Internet Fraud tendency & 986 & 76.60 & 17.88 \\
\hline Self Control & 986 & 37.53 & 7.65 \\
\hline Materialistic Value & 986 & 19.26 & 5.30 \\
\hline Peer Influence & 986 & 17.09 & 4.96 \\
\hline Impulsivity & 986 & 66.07 & 10.99 \\
\hline Age & 986 & 23.58 & 4.04 \\
\hline Sex & 986 & 1.41 & .49 \\
\hline Highest Educational Status & 986 & 3.13 & 1.12 \\
\hline
\end{tabular}

Table 1 above shows the mean and standard deviation of all the variables in the study. Internet fraud tendency has the highest mean and standard deviation ( $\bar{X}=76.60, \mathrm{SD}=17.88)$ while peer influence is the least $(\bar{X}=17.09, \mathrm{SD}=4.96)$.

\section{Test of Hypothesis}

Table 2 showing regression of the predictor and demographic variables on internet fraud tendency.

\begin{tabular}{|c|c|c|c|c|c|c|c|}
\hline Variables & $\beta$ & $T$ & sig & $\mathbf{R}$ & $\mathbf{R}^{2}$ & $\mathrm{~F}$ & $\mathbf{P}$ \\
\hline Self control & -.010 & -.359 & $>.05$ & \multirow{7}{*}{.479} & \multirow{7}{*}{.229} & \multirow{7}{*}{40.684} & \multirow{7}{*}{$<.001$} \\
\hline Materialistic Value & -.096 & -2.97 & $<.01$ & & & & \\
\hline Peer Influence & -.396 & -12.24 & $<.001$ & & & & \\
\hline Impulsivity & -.007 & -.257 & $>.05$ & & & & \\
\hline Age & -.095 & -3.21 & $<.01$ & & & & \\
\hline Sex & .11 & 3.55 & $<.01$ & & & & \\
\hline Highest Education & .002 & .078 & $>.05$ & & & & \\
\hline
\end{tabular}


From the table 2 above, all the variables jointly predicted internet fraud tendency among youths in Lagos and Ibadan $F(7$, $965)=40.684 .76, P<.001$. The multiple $R$ result obtained was 0.48 with $R^{2}$ of 0.23 . They jointly contributed $23 \%$ of the variance in internet fraud tendency. The independent prediction of the variables among the psychological factors showed that peer influence and materialistic value had significant predictions on internet fraud tendency $(\beta=-.096, \quad t=-2.97$, $p<.01 ; \beta=-.396 t=-12.24, p<.001)$. While, among the demographic factors, Age and sex were significant predictors of internet fraud tendency $(\beta=-.095, t=-3.21, \quad p<.01 ; \beta=.11, t=3.55, p<.01)$. However, Self-control $(\beta=-.010, t=-.359$, $p>.05)$, impulsivity $(\beta=-.007, t=-.257, p>.05)$, highest education $(\beta=-.002, t=.078, p>.05)$ did not significantly predict internet fraud tendency among youths in Lagos and Ibadan.

Table 3: Hierarchical Regression Showing The Influence Of Psychological And Demographic Variables On Internet Fraud Tendency Among Youths In Ibadan And Lagos

\begin{tabular}{|c|c|c|}
\hline PREDICTORS & MODEL 1 & MODEL 2 \\
\hline Peer influence & $.441^{\star *}$ & $.401^{\star *}$ \\
Materialistic & & $.084^{\star}$ \\
Impulsivity & & -.024 \\
Self - Control & & -.009 \\
Age & & .105 \\
Sex & & -.095 \\
Highest Education & & .448 \\
$\mathrm{R}$ & $.441^{\star *}$ & $.447^{\star *}$ \\
$\mathrm{R}^{2}$ & $.195^{\star *}$ & $.200^{\star *}$ \\
$\Delta \mathrm{R}^{2}$ & $.194^{\star *}$ & $.198^{\star \star}$ \\
$\mathrm{Df}$ & 984 & 983 \\
$\mathrm{~F}$ & 237.758 & 122.967 \\
\hline${ }^{*} \mathrm{p}<.05 ;{ }^{* *} \mathrm{p}<01$ & &
\end{tabular}

Table.3 above reveal that when the first model was tested, peer influence predicted $19.5 \%$ observed in internet fraudulent tendency $\left(R^{2}=.20, F=237.76, d f=1, p<.01\right)$. When materialistic value and other factors were added to the internet fraud tendency model, the model predicted $0.5 \%$ change observed in internet fraudulent tendency of youths $\left(R^{2}=\right.$ $.200, \Delta R^{2}=.19, F=122.97, d f=2, p<.01$ ) increasing the effect of the psychological and demographic model. Conclusively, peer influence is the major predictor of internet fraud tendency. Materialistic value, age and sex only contributed $0.5 \%$ of the change noticed in the prediction while the other factors had no significant contribution.

\section{Discussion}

The main purpose of this study was to contribute to the growing body of knowledge on internet fraud and some salient tendencies that might predispose youths to perpetuate the behaviour. In this study therefore, numerous variables identified in literature by past researchers with capacity to influence internet fraud tendency were investigated at the various stages of the study and their level of influence were statistically determined.

The tested hypothesis state that psychological factors such as self-control, peer influence, materialistic values, impulsivity and demographic factors such as age, sex and education will significantly independently and jointly predict self-reported internet fraud tendencies among youths in Lagos and Ibadan. The result showed that all the variables jointly predicted internet fraud tendency. This finding is in agreement with Wright and Collar (2000) who found that occupational crime and delinquency are related both to internal factors mediating criminal propensities as well as to external factors such as interaction with other delinquency. At their individual prediction, age, sex, materialistic value and peer influence were significant while peer influence had the greatest prediction. Burroughs and Rindfleisch (2002) reported a similar finding in their study. They reported that high level of materialist value which has been found to mediate between the individual's orientation towards material values and a collective orientation towards family and religious values. The clamour of youths for material acquisition is one of the factors that further predispose them to internet fraud. The influence of peers on internet fraud behaviour was equally noted by Piquero, et al (2005), Atwai (2011) and Holt (2011). They observed that associating with delinquent peers, is an important predictor of delinquent behaviour among youths. This result was similar to the findings of Burton et al. (1999) who observed the effect of imprudent behaviour among youths. The import of their findings is that the older a youth is, the more the internet fraud behaviour such youth manifest. It is also worthy of note that irrespective of whether a youth's parent is educated or not youths could still engage in internet fraud. The sex of a youth also has a role to play in the adoption of internet fraud behaviour. This is in agreement 
with many literatures on crime and crime diaries. Males are predominantly more involve in crimes than females as confirmed in this study.

In conclusion, peer influence, self-control, materialistic values and impulsivity were significant predictors of internet fraudulent tendency. However, from the various results it is imperative those youths who are more susceptible to peer influence, that places high premium for materialism and are impulsive has greater tendency to perpetuate internet fraud. In some cases such youths may either be involved in such behaviour or are likely involved in other types of fraudulent conducts. However, the factors identified in this study are not exhaustive as there are still other factors that could predispose youths towards internet fraud tendency. Nonetheless, this study is a bold step towards understanding youths that will likely venture into such fraudulent activities. Therefore for an effective control of fraudulent activities among the youth populace particularly on the internet, more research is required to understand the various factors responsible for the increase of such behaviour among them.

\section{References}

Adeniran, A. (2006). A Non-Dependent Frameworkfor Development, This day, www.thisdayo!1line.com

Atwai (2011)Atwai A. (2011) Youth Cybercrime Influenced by Peers Youth Today.University of Liverpool.http://www.youthtoday.org/ viewarticle.cfm?article id $=4859$

Belk, R. W. (1983) Worldly possessions: Issues and criticisms, in Advances in Consumer Research, 10, eds. R.P. Bagozzi, A.M. Tybout, Ann Arbor: Association for Consumer Research, pp.514-519.

Blackburn, R. (1995) The Psychology of Criminal Conduct. Theory, Research and Practice. John Wiley and Sons. Chis chester, England. 235-247.

Buchanan, J. (2001) Investigating and prosecuting Nigerian Fraud.United State Attorneys' Bulletin, Nov.2001.

Burton, V.S. Jr., Evans, TD. Cullen, G.T Olivares, K.M. \& Dunaway( 1999) Age, self -Control, Operationalization Theories and Adult Criminality, Journal of' Qualitative Criminology 10. 213-239.

Chan, K and Zhang, C. (2007)Living in a Celebrity-mediated social world: the Chinese experience. Young Consumers, 8(2),139-152.

Chua C. E. H. \& Wareham, J. (2002). Self regulating for on-line Auctions: An Analysis Paper presented at the International conference on Information System. Barcelona, Spain. 17-18.

Chua C.E. H. \& Wareham, J. (2004).Parasitism in internet fraud Ecologies. Dept. of Computer Information Systems: J Mack Robinson College of Business, Georgia State University,USA. 9-16.

Coombs, R. H., Paulson, M. J., \& Richardson, M. A. (1991).Peer V. S. parental influence in substance use among Hispanic and Anglo children and adolescents. Journal of Youth and Adolescence, 20, 73-88.

Easterlin, Richard A., Crimmins, Eileen M. (1991)Private Materialism, Personal Self-Fulfillment, Family Life, and Public Interest: The Nature, Effects, and Causes of Recent Changes in the Values of American Youth. Public Opinion Quarterly. 55, (4), 499-533.

Forster, D.R (2004). Can the General Theory of Crime Account for Computer Offenders:Testing Low self-Control: As a Predictor of Computer Crime Offending. Unpublished Thesis. University of Maryland, College Park, USA 167

Goldberg, M.E., Gorn, G.J., Peracchio, L.A., \&Bomossy, G. (2003).Materialism among youth.Journal of Consumer Psychology, 13(3), $278-288$.

Gottfredson M. R and Hirschi,T (1990).A general theory of crime. Stanford, CA: Stanford University Press.

Grazioli, S \&Javenpa, S.L. (2003) Deceived: Under Target Online. Communication of ACM, 46 (12): 196-205.

Holt T.J (2011) Low Self-Control, Deviant Peer Associations, and Juvenile Cybercrime. American Journal of Criminal Justice, News.msu.edu/medial. . .06/963e40c7-08ff-411d-af1-fe7563496f89.pdf

Jackson E.P. Tittle C.R. \& Burke, M. (1985). Cited in Virginia Brookes "what turn so called Ordinary Citizens into Ruthless Killers". Science in Africa. Online Magazine. http/lwww.scienceinafrica.co.za

Kasser, Ryan, Couchman and Sheldon (2004) Kasser, T., Ryan, R.M., Couchman, C.E. \& Sheldon, K. M. (2004) Materialistic values: Their causes and consequences, in T. Kasser\& A.D. Kanner (eds.), Psychology and Consumer Culture, Washington, D.C.: American Psychology Association.

Mangleburg T.F. \& Bristol, T (1998) Socialization and adolescents' Skepticism towards advertising.Journal of advertising, 27(3), 11-21.

Osimiri, U. 1997, "Appraisal of Nigerian AdvanceFee Fraud Legislation 1995", Journal of Financial Crime, vol. 4, no. 3, pp. $271-277$.

Richins M L \& Dawson D. (1992) A consumer Values Orientation for materialism an its measurement: scale development and validation. Journal of Consumer Research, 19(December) 303-316.

Richins'M.L (2004). The Material value scales: measurement properties and development of a short form. Journal of Consumer Research. 31(1), 209-219.

Rogers,M Seigfried K and Tidke, K (2006) Self-reported computer criminal behavior: A psychological analysis. Proceedings of the Digital ForensicsWorkshop.Elseiverpp. 116-120.

Saunders, S. (2001). Fromm's marketing character and Rokeach values. Social Behavior and Personality, 29(2), 191-196.

Tanfa, D.Y. (2006) Advance Fee Fraud. Doctoral Thesis. University of South Africa (Criminology) httpllwww/google.com.ng/search?q= Tanfa+ 2006\&h1=en\&start= 10\&Sa=N

Tangney, J.P. Baumeister, R.F. and Boone, A.L.(2004). High self Control Predicts Good Adjustment, Less Pathology, Better Grades and Interpersonal Success. Journal of Personality 72,2,271-295. 